Lactobacillus plantarum 69-2 combined with galacto-oligosaccharides alleviates D-galactose-induced aging by regulating AMPK/SIRT1 signaling pathway and the gut microbiota in mice

Authors: Wan Wang ${ }^{1,2}$, Fei Liu ${ }^{1,2}$, Cong Xu ${ }^{1,2}$, Zhijing Liu ${ }^{1,2}$, Jiage Ma ${ }^{1,2}$, Liya Gu ${ }^{1}$,

${ }^{2}$, Zhanmei Jiang ${ }^{1,2^{*}}$ and Juncai Hou ${ }^{1,2 *}$

\title{
Affiliation :
}

${ }^{1}$ College of Food Science, Northeast Agricultural University, Harbin, 150030, China

${ }^{2}$ Key Laboratory of Dairy Science, Ministry of Education, College of Food Science, Northeast Agricultural University, Harbin 150030, China

* Corresponding authors:

Juncai Hou, Tel: 0086-451-55190459; Fax: 0086-451-55190577; E-mail: jchou@neau.edu.cn

Zhanmei Jiang, Tel: 0086-451-55190459; Fax: 0086-451-55190577; E-mail: zhanmeijiang@neau.edu.cn 


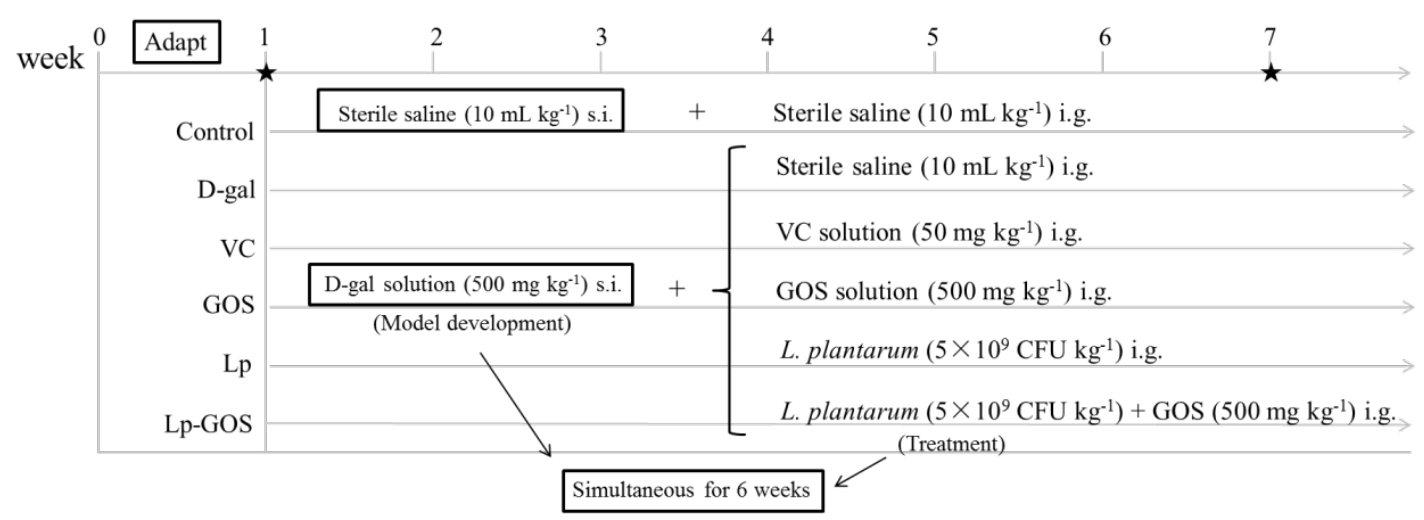

Figure S1 Design of animal experiment. s.i.: Subcutaneous injection; i.g.: Intragastric gavage. Intragastric administration was given $30 \mathrm{~min}$ after subcutaneous injection once a day for 6 weeks. 
Table S1 Molecular Formula and Abundance of GOS with Different Degree of

\section{Polymerization}

\begin{tabular}{|c|c|c|}
\hline Degree of polymerization & Structure of GOS & Abundance $(\mathrm{mol} / \mathrm{mol})$ \\
\hline \multirow{5}{*}{ Trisaccharide } & $\beta$-D-Gal-( $1 \rightarrow 6)-\beta$-D-Gal-( $(1 \rightarrow 6)-\mathrm{D}-\mathrm{Gal}$ & \multirow{5}{*}{$14.03 \%$} \\
\hline & $\beta$-D-Gal-( $1 \rightarrow 6)-\beta$-D-Gal-( $(1 \rightarrow 4)-\mathrm{D}-\mathrm{Glc}$ & \\
\hline & $\beta$-D-Gal-( $(1 \rightarrow 4)-\beta$-D-Gal-( $(1 \rightarrow 4)$-D-Glc & \\
\hline & $\beta$-D-Gal-( $1 \rightarrow 4)-\beta$-D-Gal-( $(1 \rightarrow 6)$-D-Glc & \\
\hline & $\beta$-D-Gal-( $1 \rightarrow 6)-\beta$-D-Gal- $(1 \rightarrow 6)-\mathrm{D}-\mathrm{Glc}$ & \\
\hline \multirow{3}{*}{ Tetrasaccharide } & $\beta$-D-Gal-( $1 \rightarrow 4)-\beta$-D-Gal-( $(1 \rightarrow 4)-\beta$-D-Gal-( $1 \rightarrow 4)$-D-Glc & \multirow{3}{*}{$19.91 \%$} \\
\hline & $\beta$-D-Gal-( $1 \rightarrow 6)-\beta$-D-Gal-( $(1 \rightarrow 6)-\beta$-D-Gal-( $1 \rightarrow 4)$-D-Glc & \\
\hline & $\beta$-D-Gal-( $(1 \rightarrow 4)-\beta$-D-Gal-( $(1 \rightarrow 4)-\beta$-D-Gal-( $(1 \rightarrow 6)$-D-Glc & \\
\hline \multirow{4}{*}{ Pentasaccharide } & $\beta$-D-Gal-( $(1 \rightarrow 6)-\beta$-D-Gal-( $(1 \rightarrow 6)-\beta$-D-Gal-( $(1 \rightarrow 6)-\beta$-D-Gal-( $(1 \rightarrow 4)$-D-Glc & \multirow{4}{*}{$68.06 \%$} \\
\hline & $\beta$-D-Gal-(1 $\rightarrow 4)-\beta$-D-Gal-( $1 \rightarrow 4)-\beta$-D-Gal-( $1 \rightarrow 6)-\beta$-D-Gal-( $1 \rightarrow 4)$-D-Glc & \\
\hline & $\beta$-D-Gal-( $(1 \rightarrow 4)-\beta$-D-Gal-( $(1 \rightarrow 4)-\beta$-D-Gal-( $1 \rightarrow 4)-\beta$-D-Gal-( $(1 \rightarrow 6)$-D-Glc & \\
\hline & $\beta$-D-Gal-( $(1 \rightarrow 4)-\beta$-D-Gal-( $1 \rightarrow 4)-\beta$-D-Gal-( $(1 \rightarrow 4)-\beta$-D-Gal-( $(1 \rightarrow 4)$-D-Glc & \\
\hline
\end{tabular}

${ }^{a} \beta$-D-Gal- $(1 \rightarrow \mathrm{n})$ meant $\mathrm{D}$-galactosyl, and its $\beta$-glycoside linkage site is on the $\mathrm{n}$-point of the next galactosyl

b -D-Gal meant the terminal D-galactosyl; -D-Glc meant the terminal D-glucosyl 
Table S2 Characteristics Tests of Lactobacillus

\begin{tabular}{ccccc}
\hline & $\begin{array}{c}\text { Simulated gastric } \\
\text { digestion }\end{array}$ & $\begin{array}{c}\text { Simulated intestinal } \\
\text { digestion }\end{array}$ & $\begin{array}{c}\text { Cell adhesion } \\
\text { ability }\end{array}$ & $\begin{array}{c}\text { Surface } \\
\text { hydrophobicity }\end{array}$ \\
\hline L. plantarum 56 & $(35.58 \pm 1.32) \%^{\mathrm{c}}$ & $(30.18 \pm 0.88) \%^{\mathrm{c}}$ & $(61.59 \pm 2.31) \%^{\mathrm{b}}$ & $(42.32 \pm 1.74) \%^{\mathrm{d}}$ \\
L. plantarum 59 & $(40.46 \pm 1.85) \%^{\mathrm{a}}$ & $(48.76 \pm 0.99) \%^{\mathrm{b}}$ & $(66.51 \pm 1.84) \%{ }^{\mathrm{b}}$ & $(39.17 \pm 0.79) \%^{\mathrm{e}}$ \\
L. plantarum 69-2 & $(38.28 \pm 0.80) \%^{\mathrm{b}}$ & $(48.90 \pm 1.44) \%^{\mathrm{b}}$ & $(75.50 \pm 0.84) \%{ }^{\mathrm{a}}$ & $(60.23 \pm 2.42) \%^{\mathrm{b}}$ \\
L. rhamnosus 1.0320 & $(36.21 \pm 0.44) \%^{\mathrm{c}}$ & $(28.73 \pm 1.48) \%^{\mathrm{c}}$ & $(78.92 \pm 1.49) \%^{\mathrm{a}}$ & $(68.64 \pm 1.99) \%^{\mathrm{a}}$ \\
L. plantarum 1.0628 & $(21.43 \pm 1.31) \%^{\mathrm{d}}$ & $(52.49 \pm 1.27) \%^{\mathrm{a}}$ & $(48.67 \pm 2.52) \% \mathrm{c}$ & $(52.38 \pm 2.10) \%^{\mathrm{c}}$ \\
\hline
\end{tabular}

a The simulated gastric digestion test was incubated in the simulated gastric fluid (pepsin: $3 \mathrm{~g} / \mathrm{L}, \mathrm{pH} 2.0$ ) for $180 \mathrm{~min}$.

${ }^{\mathrm{b}}$ The simulated intestinal digestion test was incubated in the simulated intestinal fluid (pancreatin: $1 \mathrm{~g} / \mathrm{L}$ and bile salts: $4.5 \mathrm{~g} / \mathrm{L}$ ) for $180 \mathrm{~min}$.

${ }^{\mathrm{c}}$ All data are indicated as mean $\pm \mathrm{SD}$ of 3 times per group

${ }^{\mathrm{d}}$ Different letters represent significant differences in the same column $(P<0.05)$ 
Table S3 Antimicrobial Susceptibility Test of Lactobacillus

\begin{tabular}{cccccc}
\hline \multirow{2}{*}{ Antibiotics } & L. plantarum 56 & L. plantarum 59 & $\begin{array}{c}\text { L. plantarum } \\
69-2\end{array}$ & L. rhamnosus 1.0320 & L. plantarum 1.0628 \\
\cline { 2 - 5 } & \multicolumn{3}{c}{ Bacterial inhibition zones $(\mathrm{mm}) /$ Antimicrobial susceptibility determination } \\
\hline Ampicillin & $30.25 \pm 2.13 / \mathrm{S}$ & $26.73 \pm 0.98 / \mathrm{R}$ & $40.55 \pm 1.33 / \mathrm{S}$ & $34.75 \pm 1.53 / \mathrm{S}$ & $27.07 \pm 0.57 / \mathrm{R}$ \\
Cefalexin & $13.39 \pm 0.78 / \mathrm{R}$ & $10.98 \pm 1.21 / \mathrm{R}$ & $42.33 \pm 0.19 / \mathrm{S}$ & $18.33 \pm 1.24 / \mathrm{S}$ & $38.52 \pm 072 / \mathrm{S}$ \\
Gentamicin & $0.00 \pm 0.00 / \mathrm{R}$ & $8.52 \pm 0.73 / \mathrm{R}$ & $14.67 \pm 1.35 / \mathrm{I}$ & $18.15 \pm 1.55 / \mathrm{S}$ & $11.67 \pm 0.97 / \mathrm{R}$ \\
streptomycin & $0.00 \pm 0.00 / \mathrm{R}$ & $0.00 \pm 0.00 / \mathrm{R}$ & $17.66 \pm 0.58 / \mathrm{S}$ & $16.34 \pm 0.26 / \mathrm{S}$ & $0.00 \pm 0.00 / \mathrm{R}$ \\
Erythromycin & $18.86 \pm 0.91 / \mathrm{I}$ & $17.80 \pm 0.58 / \mathrm{I}$ & $33.39 \pm 0.69 / \mathrm{S}$ & $31.67 \pm 0.31 / \mathrm{S}$ & $30.33 \pm 0.29 / \mathrm{S}$ \\
Oxytetracycline & $16.02 \pm 0.87 / \mathrm{S}$ & $17.95 \pm 1.13 / \mathrm{S}$ & $28.43 \pm 2.54 / \mathrm{S}$ & $23.44 \pm 1.87 / \mathrm{S}$ & $19.96 \pm 1.54 / \mathrm{S}$ \\
Ciprofloxacin & $18.65 \pm 1.37 / \mathrm{I}$ & $20.59 \pm 0.74 / \mathrm{I}$ & $54.57 \pm 1.37 / \mathrm{S}$ & $21.48 \pm 0.57 / \mathrm{S}$ & $0.00 \pm 0.00 / \mathrm{R}$ \\
Tetracycline & $16.28 \pm 0.96 / \mathrm{I}$ & $16.56 \pm 0.94 / \mathrm{I}$ & $25.67 \pm 0.42 / \mathrm{S}$ & $24.37 \pm 1.55 / \mathrm{S}$ & $25.37 \pm 1.83 / \mathrm{S}$ \\
Chloramphenicol & $0.00 \pm 0.00 / \mathrm{R}$ & $0.00 \pm 0.00 / \mathrm{R}$ & $32.93 \pm 1.39 / \mathrm{S}$ & $19.23 \pm 1.19 / \mathrm{S}$ & $33.80 \pm 0.53 / \mathrm{S}$ \\
Vancomycin & $0.00 \pm 0.00 / \mathrm{R}$ & $0.00 \pm 0.00 / \mathrm{R}$ & $0.00 \pm 0.00 / \mathrm{R}$ & $0.00 \pm 0.00 / \mathrm{R}$ & $0.00 \pm 0.00 / \mathrm{R}$ \\
\hline
\end{tabular}

${ }^{\text {a }}$ S: Susceptible; I: Intermediate; R: Resistance

${ }^{\mathrm{b}}$ All data are indicated as mean $\pm \mathrm{SD}$ of 3 times per group 
Table S4 Total Variance Explained and Antioxidant Capacity Score by Principal Component Analysis

\begin{tabular}{ccccccc}
\hline \multirow{2}{*}{ Component } & \multicolumn{3}{c}{ Initial Eigenvalues } & \multicolumn{2}{c}{ Extraction Sums of Squared Loadings } \\
\cline { 2 - 6 } & Total & \% of Variance & Cumulative \% & Total & \% of Variance & Cumulative \% \\
\hline 1 & 2.017 & 67.220 & 67.220 & 2.017 & 67.220 & 67.220 \\
2 & 1.020 & 28.668 & 95.888 & 1.020 & 28.668 & 95.888 \\
3 & 0.123 & 4.112 & 100.000 & & & \\
\hline Lactobacillus Strain & F1 Score & F2 Score & F Total Score & Sequence \\
\hline L.plantarum 56 & -0.3833 & 0.695564 & -0.06074 & 4 \\
L.plantarum 59 & -0.02415 & 1.137197 & 0.323061 & 2 \\
L.plantarum 69-2 & 1.857078 & -0.32377 & 1.205061 & 1 \\
L.rhamnosus 1.0320 & -0.81002 & -1.6734 & -1.06815 & 6 \\
L.plantarum 1.0628 & -0.83794 & 0.474601 & -0.44553 & 5 \\
L.rhamnosus GG & 0.198346 & -0.31019 & 0.046307 & 3 \\
\hline
\end{tabular}


Table S5 Data Statistics of High-throughput Sequencing of Cecum Contents

\begin{tabular}{ccccccccc}
\hline Sample & Clean paired reads & GC(\%) & Q30(\%) & Num seqs & Sum len & Min len & Avg len & Max len \\
\hline Control1 & 64521 & $53 \%$ & $96.51 \%$ & 55,056 & $22,989,980$ & 402 & 417.6 & 433 \\
Control2 & 68268 & $54 \%$ & $95.66 \%$ & 57,396 & $23,800,085$ & 401 & 414.7 & 432 \\
Control3 & 72869 & $53 \%$ & $95.83 \%$ & 61,333 & $25,507,119$ & 401 & 415.9 & 433 \\
D-gal1 & 70225 & $52 \%$ & $96.14 \%$ & 59,426 & $24,886,774$ & 401 & 418.8 & 445 \\
D-gal2 & 76326 & $53 \%$ & $96.48 \%$ & 64,943 & $27,148,219$ & 403 & 418 & 445 \\
D-gal3 & 71019 & $52 \%$ & $96.12 \%$ & 60,168 & $25,349,535$ & 403 & 421.3 & 447 \\
GOS1 & 65071 & $54 \%$ & $95.20 \%$ & 54,139 & $22,593,166$ & 403 & 417.3 & 445 \\
GOS2 & 54655 & $53 \%$ & $95.74 \%$ & 46,012 & $19,134,716$ & 403 & 415.9 & 433 \\
GOS3 & 83354 & $53 \%$ & $96.09 \%$ & 70,375 & $29,325,986$ & 401 & 416.7 & 433 \\
Lp1 & 59220 & $53 \%$ & $95.38 \%$ & 49,993 & $20,789,874$ & 401 & 415.9 & 445 \\
Lp2 & 69902 & $53 \%$ & $95.84 \%$ & 58,837 & $24,508,959$ & 401 & 416.6 & 446 \\
Lp3 & 69428 & $53 \%$ & $96.37 \%$ & 59,124 & $24,622,935$ & 402 & 416.5 & 445 \\
Lp-GOS1 & 63260 & $53 \%$ & $96.28 \%$ & 53,610 & $22,320,843$ & 403 & 416.4 & 431 \\
Lp-GOS2 & 57434 & $52 \%$ & $96.03 \%$ & 48,589 & $20,274,017$ & 401 & 417.3 & 445 \\
Lp-GOS3 & 76149 & $53 \%$ & $95.64 \%$ & 63,691 & $26,668,555$ & 403 & 418.7 & 437 \\
\hline
\end{tabular}

${ }^{\text {a }} \mathrm{GC}(\%)$ : the percentage of the sum of $\mathrm{G}$ and $\mathrm{C}$ in the total bases

${ }^{\mathrm{b}} \mathrm{Q} 30$ (\%): the percentage of bases with Phred values greater than 20 and 30 in the total bases. Phred $=-10 \lg \mathrm{E}, \mathrm{E}$ is the error rate

c Num seqs: sequences number; Sum len: sum of length; Min len: minimum length;

Avg len: average length; Max len: maximum length 